\title{
ACTUATOR DESIGN FOR ARC WELDING ROBOT
}

\author{
${ }^{1}$ Anurag Verma, ${ }^{2}$ M. M. Gor* \\ ${ }^{1}$ G.H Patel College of Engineering \& Technology, V.V.Nagar-388120, Gujarat, India \\ ${ }^{2}$ Parul Institute of Engineering \& Technology, Limda-391760, Gujarat, India \\ *Corresponding author: mehulmgor@ rediffmail.com \\ Received 21 January, 2010; Revised 11 June, 2010
}

\begin{abstract}
The present work is an attempt to determine torque required at each joint of 6-Degree of Freedom Arc Welding Robot for typical horizontal fillet welding operation which will be useful for designing actuator. Here, range of welding speed $90 \mathrm{~mm} / \mathrm{min} \& 2000 \mathrm{~mm} / \mathrm{min}$ is taken. Result obtained at the end of this analysis will be useful for designing actuator capacity for typical application. The methodology adopted for analysis includes development of dynamic model, determination of velocity and acceleration with respect to time and thus determination of torque using velocity-acceleration and dynamic model.
\end{abstract}

Keywords : Inverse Dynamic Analysis, Lagrange-Euler formulation, Arc Welding Robot

\section{INTRODUCTION}

Robot arm dynamics deals with the mathematical formulations of the equations of robot arm motion $^{7}$. The dynamic equations of motion of a manipulator are a set of mathematical equations describing the dynamic behavior of the manipulator. Such equations of motion are useful for computer simulation of the robot motion, the design of suitable control equations for a robot arm, and the evaluation of the kinematics design and structure of a robot arm. The dynamic behavior of the manipulator provides relationship between joint actuator torques and motion of links for simulation and design of control algorithms. The forward dynamics ${ }^{8}$ deals with determination of generalized accelerations $\ddot{q}(t)$ and thus, $\dot{q}(t)$ and $q(t)$, for the generalized input torque $\tau(\mathrm{t})$ for $t>t_{0}$. The inverse dynamics problem ${ }^{8}$ is of great importance to robot control and programming because it allows one to find the inputs necessary for producing the desired outputs.

To perform desired work robot's end effector has to follow the specific trajectory. To do so all the joints and thus links will be operated with respect to time. And all the joints actuator should be in position to transmit enough torque. For arc welding operation, robot has to follow a predefined trajectory, so it is necessary to know torque required to operate each joint for selected trajectory path. The present work is an attempt to find out torque required at each joint of 120iBe Fanuc 6-Degree of Freedom Arc Welding Robot for $450 \mathrm{~mm}$ horizontal fillet welding operation as shown in Figure 1. Here maximum and minimum welding speed is considered through which range of torque requirement is calculated, which will be helpful for the actuator selection. 


\section{MATERIALS AND METHODS}

To find out torque, dynamic model should be generated. Then velocity and acceleration are determined for range of welding speed. Using velocity \& acceleration, dynamic model is solved and torque is determined.

\section{Dynamic Model}

The derivation of the dynamic model of a manipulator based on the Lagrange-Euler formulation is simple and systematic ${ }^{7}$. Assuming rigid body motion, the resulting equations of motion, excluding the dynamics of electronic control devices, back lash and gear friction, are a set of second-order coupled nonlinear differential equations.

To derive dynamic model, technical specification of robot and real application specification are required, based on which frame assignment is carried out. From assigned frame, DH parameter, Centroidal and gravity direction is finalized. Using all these, dynamic model is generated by Lagrange-Euler Formulation.

\section{Technical specification of 120iBe Fanuc Robot \& Real Application Specification}

$120 \mathrm{iBe}$ Fanuc Arc Welding robot is used by Jyoti Automation, Rajkot, India for manufacturing of $\mathrm{CNC}$ body parts. $120 \mathrm{iBe}$ is a 6 Degree of Freedom robot having all rotary joint and maximum joint rotation and maximum speed as per Table 1.

Also it is mentioned that this analysis is carried out for typical application i.e. $450 \mathrm{~mm}$ horizontal fillet welding operation as shown in Figure 1, the joint rotation required for the said task will be as per Table 2 .
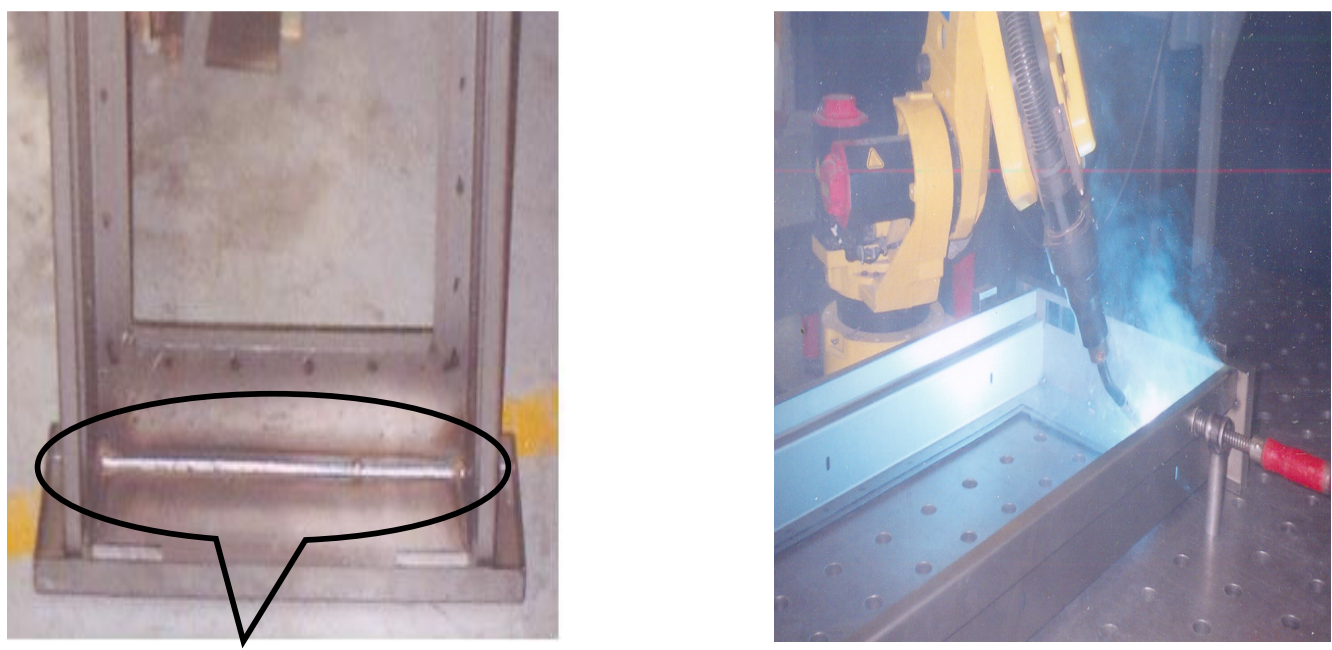

$450 \mathrm{~mm}$ horizontal fillet welding

Figure 1. Typical Application: $450 \mathrm{~mm}$ horizontal fillet weld by Robot 
Table 1 Technical Specification of Robot $^{5}$

\begin{tabular}{|c|c|c|}
\hline Jt. & $\begin{array}{c}\text { Rotation } \\
\left(\text { rad }^{5}\right.\end{array}$ & $\begin{array}{c}\text { Max. Speed } \\
\left(\text { rad/s }^{5}\right.\end{array}$ \\
\hline J1 & 5.99 & 2.88 \\
\hline J2 & 4.36 & 2.88 \\
\hline J3 & 8.03 & 3.05 \\
\hline J4 & 6.98 & 6.11 \\
\hline J5 & 4.89 & 5.93 \\
\hline J6 & 15.7 & 9.08 \\
\hline
\end{tabular}

Table 2 Rotation of each joint for typical application

\begin{tabular}{|c|c|c|c|}
\hline Jt. & $\begin{array}{c}\text { Initial } \\
\text { Position }\end{array}$ & Final Position & $\begin{array}{c}\text { Rotation } \\
\text { for Weld }\end{array}$ \\
\hline J1 & $80.64^{0}$ & $68.06^{0}$ & $-12.57^{0}$ \\
\hline J2 & $18.13^{0}$ & $9.39^{0}$ & $-8.73^{0}$ \\
\hline J3 & $-58.72^{0}$ & $-58.56^{0}$ & $0.16^{0}$ \\
\hline J4 & $-62.96^{0}$ & $-30.29^{0}$ & $32.67^{0}$ \\
\hline J5 & $-16.38^{0}$ & $-51.06^{0}$ & $-34.68^{0}$ \\
\hline J6 & $-47.15^{0}$ & $6.38^{0}$ & $53.53^{0}$ \\
\hline
\end{tabular}

\section{Frame Assignment}

The Denavit-Hartenberg convention is commonly used to select the coordinate frames for formulating the kinematic problem of serial manipulator ${ }^{2}$. Using the link frame assignment algorithm ${ }^{8}$ first of all the $\mathrm{Z}_{\mathrm{i}}$-axis of the entire joint is fixed in the direction of rotation as followed by the right-handed rule for rotation. The index number for the Z-axis of joint $n$ in each case is $n-1$. That means, joint 1 rotation axis is denoted as $Z_{0}$. Here the rotation about the $\mathrm{Z}$-axis will be the joint variable. Then $\mathrm{X}_{\mathrm{i}}$ axis is fixed considering $\mathrm{Z}_{\mathrm{i}-1}$ and $\mathrm{Z}_{\mathrm{i}}$ axes. And finally $\mathrm{Yi}$ axis is fixed to complete the right handed ortho-normal coordinate frame $\{\mathrm{i}\}$.

\section{DH parameter, Centroidal \& Gravity Direction}

From the assigned frame, DH Parameter ${ }^{6,7,8,9,10}$ i.e. four joint-link parameters a, $\alpha, d, \theta$ for each link are finalized as shown in Table 3. Also centroidal position and gravity direction ${ }^{8}$ are finalized as shown in Table 4. Description of each symbol is as per Appendix-1

Table 3 DH parameter

\begin{tabular}{|c|c|c|c|}
\hline $\begin{array}{c}a \\
(m)\end{array}$ & $\begin{array}{c}\alpha \\
(\mathrm{deg})\end{array}$ & $\begin{array}{c}d \\
(\mathrm{~m})\end{array}$ & $\begin{array}{c}\theta \\
(\mathrm{deg})\end{array}$ \\
\hline 0.150 & -90 & 0.300 & $\theta_{1}$ \\
\hline 0.770 & 0 & 0 & $90+\theta_{2}$ \\
\hline 0.100 & -90 & 0.150 & $\theta_{3}$ \\
\hline 0 & 90 & 0.740 & $\theta_{4}$ \\
\hline 0.050 & -90 & 0 & $\theta_{5}$ \\
\hline 0 & 0 & 0.300 & $\theta_{6}$ \\
\hline
\end{tabular}

Table 4 Centroidal \& Gravity Direction

\begin{tabular}{|c|l|c|}
\hline Frame & Centroidal Position & $\begin{array}{l}\text { Gravity } \\
\text { Direction }\end{array}$ \\
\hline 1 & {$\left[\begin{array}{llll}0.075 & 0.150 & 0 & 1\end{array}\right]$} & $-\mathrm{Z}$ \\
\hline 2 & {$\left[\begin{array}{llll}0.385 & 0 & 0 & 1\end{array}\right]$} & $\mathrm{Y}$ \\
\hline 3 & {$\left[\begin{array}{lllll}0.050 & 0.075 & 0 & 1\end{array}\right]$} & $\mathrm{X}$ \\
\hline 4 & {$\left[\begin{array}{lllll}0 & -0.370 & 0 & 1\end{array}\right]$} & $\mathrm{X}$ \\
\hline 5 & {$\left[\begin{array}{lllll}0.025 & 0 & -0.050 & 1\end{array}\right]$} & $\mathrm{X}$ \\
\hline 6 & {$\left[\begin{array}{llll}0 & 0 & -0.150 & 1\end{array}\right]$} & $\mathrm{X}$ \\
\hline
\end{tabular}

\section{Dynamic model in terms of Velocity \& Acceleration}

To get the mass of each link of robot its dynamic model based on Lagrange-Euler formulation $^{1,8}$ is used. Here indigenous MATLAB program is developed to derive dynamic model of the robot in terms of mass, velocity and acceleration. Output of dynamic model program will be the 6 equations of motion.

$\tau_{i}=\sum_{j=1}^{n} M_{i j} \ddot{q}_{j}+\sum_{j=1}^{n} \sum_{k=1}^{n} h_{i j k} \dot{q}_{j} \dot{q}_{k}+G_{i} \quad$ where $i=1$ to 6

Description of each symbol is as per Appendix 1 


\section{Velocity and Acceleration at every instant for selected arc welding operation.}

Here initial and final velocity will be zero. Also initial and final position of each joint is known. These four pieces of information allow any one to solve for unknowns by third order polynomial trajectory planning.

Therefore, to describe the joint motion, assume that the cubic polynomial ${ }^{8,10}$ is

$$
q(t)=a_{0}+a_{1} t+a_{2} t^{2}+a_{3} t^{3}
$$

which gives a parabolic velocity profile

$$
\dot{q}(t)=a_{1}+2 a_{2} t+3 a_{3} t^{2}
$$

and a linear acceleration profile

$$
\ddot{q}(t)=2 a_{2}+6 a_{3} t
$$

Using aforesaid method MATLAB program is developed which will generate position, velocity and acceleration value with time history.

\section{Torque Determination}

Dynamic model is solved by inserting value of velocity and acceleration. This dynamic model is developed through program generated in MATLAB using Lagrange-Euler formulation. Indigenous program of torque determination for selected arc welding application i.e. $450 \mathrm{~mm}$ horizontal fillet welding operation of 120iBe robot as shown in Figure 1, will accept only required time for operation, and initial and final position of each joint. Already no. of link, type of joint, DH parameter, Centroidal position, gravity direction, mass and length of each link of $120 \mathrm{iBe}$ is included in the program. Final results are determined in terms of graphs and detailed calculations are stored in text files by the developed MATLAB program $^{3,11}$.

\section{RESULTS}

To perform $450 \mathrm{~mm}$ horizontal fillet welding operation range of welding speed considered is $90 \mathrm{~mm} / \mathrm{min}$ to $2000 \mathrm{~mm} / \mathrm{min}$. According to this welding speed, arc welding operation will be completed in $300 \mathrm{sec}$ and $13.5 \mathrm{sec}$ respectively. To perform welding operation in this time limit, torque required at each joint is as per Table 4 .

Table 4 Torque at each joint for range of speed

\begin{tabular}{|c|c|c|}
\hline Joint & $\begin{array}{c}\text { Torque (N.m) for } \\
\text { welding speed } \\
90 \mathrm{~mm} / \mathrm{min}\end{array}$ & $\begin{array}{c}\text { Torque (N.m) for } \\
\text { welding speed } \\
2000 \mathrm{~mm} / \mathrm{min}\end{array}$ \\
\hline $\mathrm{J} 1$ & 50.2 & 50.6 \\
\hline $\mathrm{J} 2$ & 692 & 693 \\
\hline $\mathrm{J} 3$ & 154 & 154 \\
\hline $\mathrm{J} 4$ & 2.39 & 2.71 \\
\hline $\mathrm{J} 5$ & 8.77 & 8.81 \\
\hline $\mathrm{J} 6$ & $8.45 \times 10^{-5}$ & $4.17 \times 10^{-2}$ \\
\hline
\end{tabular}




\section{CONCLUSION}

At the end of this analysis, it is concluded that arc welding operation is very low speed operation and so velocity and acceleration will be less. Because of this low value of velocity and acceleration, torque due to inertia and centrifugal component will be low. So for this typical arc welding operation major torque is due to gravity component. This gravity component will remain constant and it will not be affected by speed. Here the problem is of inverse dynamics, the joint torque required to cause the desired motion (displacement, velocity, acceleration etc.) are computed. This analysis gives range of torque required to carry out the $450 \mathrm{~mm}$ horizontal fillet arc welding operation. Thus this work allows one to decide capacity of the actuator for typical application.

Further it is useful for manipulator trajectory planning and control algorithm implementation. The computation of torques to be applied at the joints to obtain the desired motion specified as a joint trajectory, which is specified in terms of positions, velocities, and accelerations, is useful for verifying feasibility of the desired trajectory.

During this analysis text files are generated by the program which will give complete details about the input, output and computation at various stages of the programs. Also one complete database may be prepared for the various range of selection of actuator.

\section{ACKNOWLEDGEMENTS}

The author would like to thank the Jyoti Automation, Rajkot, India for allowing this research on their arc welding robot.

\section{REFERENCES}

1. Anurag Verma and M. M. Gor 2008. "Computer Aided Dynamic Modeling of a Manipulator", Proceeding of the National Conference on Emerging Trends in Mechanical Eng., G. H. Patel College of Engg. \& Technology, V. V. Nagar, Gujarat, India.

2. Denavit, J. and R. S. Hartenberg, 1955. A kinematic notation for lower pair mechanisms. Journal of Applied Mechanics, 22: 215-221.

3. Brian R. Hunt, Ronald L. Lipsman and Jonathan M.Rosenburg 1996. A Guide to MATLAB, First Edition, Cambridge University Press, , New Delhi, India.

4. GE Fanuc Automation, AC servomotor $\beta$ is Series, Descriptions Manual

5. FANUC Robot i Series Brochure.

6. Jhon J. Craig 1986. Introduction to Robotics, Mechanics \& Control, Pearson Education.

7. K. S. Fu, R. C. Gonzalez and C.S.G. 1987. Lee, Robotics - Control, Sensing, Vision \& Intelligence, First Edition, McGraw-Hill Book Company, New Delhi, India.

8. R. K. Mittal and I. J. Nagrath 2006. Robotics \& Control, First Edition, Tata McGrawHill Publishing Co. Ltd., New Delhi, India. 
9. Robert J. Schilling, 1996. Fundamentals of Robotics, First Edition, Prentice-Hall of India Pvt. Ltd, New Delhi, India.

10. Saeed B. Niku 2003. Introduction to Robotics - Analysis, Systems \& Applications, First Edition, Pearson Education Pvt. Ltd., Delhi, India.

11. William J. Palm III 1995. Introduction to MATLAB for Engineers, First Edition, McGraw-Hill International Edition. Singapore.

\section{APPENDIX-1}

$a_{i} \quad$ Link length parameter for link i

$d_{i} \quad$ Joint distance

$G_{i} \quad$ Gravity loading vector

$h_{i j k} \quad$ Velocity induced torques vector

$M_{i j} \quad$ Manipulator inertia matrix

$n \quad$ Number of Links /joint/degree of freedom

$q(t) \quad$ Joint displacement with respect to time $\mathrm{t}$

$\dot{q}(t) \quad$ Joint velocity with respect to time $\mathrm{t}$

$\ddot{q}(t) \quad$ Joint acceleration with respect to time $\mathrm{t}$

$\tau_{i} \quad$ Joint i torque

$t \quad$ Time

$\alpha_{i} \quad$ Link twist angle for link i

$\theta_{i} \quad$ Revolute joint angle (angular displacement) 